\title{
Quantile Uncorrelation and Instrumental Regressions
}

\section{Citation}

Komarova, Tatiana, Thomas A. Severini, and Elie T. Tamer. 2012. "Quantile Uncorrelation and Instrumental Regressions." Journal of Econometric Methods 1 (1) (January 24). doi:10.1515/2156-6674.1001.

\section{Published Version}

doi:10.1515/2156-6674.1001

\section{Permanent link}

http://nrs.harvard.edu/urn-3:HUL.InstRepos:25267902

\section{Terms of Use}

This article was downloaded from Harvard University's DASH repository, and is made available under the terms and conditions applicable to Open Access Policy Articles, as set forth at http:// nrs.harvard.edu/urn-3:HUL.InstRepos:dash.current.terms-of-use\#OAP

\section{Share Your Story}

The Harvard community has made this article openly available.

Please share how this access benefits you. Submit a story.

Accessibility 


\title{
QUANTILE UNCORRELATION AND INSTRUMENTAL REGRESSIONS*
}

\author{
TATIANA KOMAROVA ${ }^{1}$, THOMAS SEVERINI $^{2}$, AND ELIE TAMER $^{3}$
}

\begin{abstract}
We introduce a notion of median uncorrelation that is a natural extension of mean (linear) uncorrelation. A scalar random variable $Y$ is median uncorrelated with a $k$-dimensional random vector $X$ if and only if the slope from an LAD regression of $Y$ on $X$ is zero. Using this simple definition, we characterize properties of median uncorrelated random variables, and introduce a notion of multivariate median uncorrelation. We provide measures of median uncorrelation that are similar to the linear correlation coefficient and the coefficient of determination. We also extend this median uncorrelation to other loss functions.

As two stage least squares exploits mean uncorrelation between an instrument vector and the error to derive consistent estimators for parameters in linear regressions with endogenous regressors, the main result of this paper shows how a median uncorrelation assumption between an instrument vector and the error can similarly be used to derive consistent estimators in these linear models with endogenous regressors. We also show how median uncorrelation can be used in linear panel models with quantile restrictions and in linear models with measurement errors.
\end{abstract}

Date: October 26, 2010.

${ }^{1}$ London School of Economics and Political Science, Department of Economics, Houghton Street, London WC2A 2AE, England. Tel.: +44-(0)20-7852-3707; fax: + 44-(0)20-7955-6592. E-mail: t.komarova@lse.ac.uk.

${ }^{2}$ Northwestern University, Department of Statistics. Support from the National Science Foundation is gratefully acknowledged.

${ }^{3}$ Northwestern University, Department of Economics. Partial support from the National Science Foundation is gratefully acknowledged.

* We thank the seminar participants at the London School of Economics, the University College London and the University of Toronto for their comments. We also appreciate feedback from the participants of the Canadian Econometric Study Group and the participants of the all UC Econometrics Conference. 


\section{INTRODUCTION}

We introduce a concept of quantile uncorrelation, or $\mathcal{L}_{1}$-uncorrelation, between two random variables that is a natural extension of the well known mean uncorrelation, or $\mathcal{L}_{2}$-uncorrelation. We term this type of uncorrelation, "median uncorrelation," which is the counterpart of the familiar mean (linear) uncorrelation, or simply uncorrelation. We characterize the relationship between random variables that are uncorrelated in this manner. We provide a series of properties that imply or are implied by median uncorrelation. Naturally, for example, independence of two random variables implies median uncorrelation (or in this case $\mathcal{L}_{p}$-uncorrelation for any $p \geq 1$ ). Also, this uncorrelation is not symmetric, and is nonadditive, but it retains an important invariance property.

We extend our definition to median uncorrelation between random vectors which results, indirectly, in a multivariate version of a quantile restriction. We also derive an asymmetric correlation measure, based on this notion of quantile uncorrelation, that takes values in $[-1,1]$ with a value of zero for uncorrelation. In addition, we provide another correlation measure that is the analog of the coefficient of determination, or $R^{2}$, in linear regressions. We also extend this concept to cover $\mathcal{L}_{p}$-uncorrelation for $p \geq 1.2$

As two stage least squares is based on exploiting linear uncorrelation between the error and an excluded random variable (the instrument), we also show that this uncorrelation leads naturally, and under easily interpretable conditions, to "instrumental" regressions with median uncorrelation. These are analogs of Basmann and Theil's two stage least squares, or 2SLS, (Theil (1953) and Basmann (1957)) as derived from the usual mean uncorrelation between two random variables. As in the classical 2SLS, median uncorrelation leads to an estimator that is derived by taking a "sample analogue" of the median uncorrelation measure. This estimator, similar to one used by Chernozhukov and Hansen (2005) (or CH), is consistent provided that this uncorrelation holds (along with other standard assumptions). Other applications are natural counterparts of existing least squares methods. For example, by exploiting this uncorrelation further, we show that as instrumental variable methods can be used in mean-based models to remedy the problem of classical measurement error, we show how variables obeying our median uncorrelation condition can be used as instruments to obtain estimates of parameters in linear models with measurement 
error under quantile restrictions. Furthermore, panel data quantile regression of differenced data delivers consistent estimates of parameters of interest without making assumptions on the individual effects under median uncorrelation restrictions. So, this uncorrelation gives support for running standard quantile regression of first differenced outcomes on first differenced regressors, under an absolute loss function to obtain consistent estimates of the slope parameters in linear models.

An important feature of the concept of median uncorrelatedness is the fact that it is defined in terms of the linear predictor, and hence is explicitly a "linear concept." Basically, it shares this property with say best linear predictors in that, heuristically, a random variable is median uncorrelated with another if the latter is not "useful" as a linear predictor of the former under absolute loss. Finally, this notion of median uncorrelation is general and is loss function based.

There is a large literature in econometrics on best predictor problems. Manski (1988) delineates estimators derived from prediction problems from various loss functions. There, best linear predictors are derived and consistent estimators are provided that are based on the analogy principle. The structural linear model based on quantile restrictions is equally well studied starting with the work of Koenker and Bassett (1978); see also Koenker (2005). There has also been a series of papers dealing with the presence of endogenous regressors in models with quantile restrictions. Amemiya (1981) proposed a two-staged least absolute deviation estimator. Then, based on method of moments, Honoré and $\mathrm{Hu}$ (2004) provide methods that can be used to do inference on parameters defined though separable moment models (that can be nonlinear). Chernozhukov and Hansen (2005) (CH) in a series of papers shed new light on general class of monotonic models with conditional quantile restrictions. They provide sufficient point identification conditions for these models, and also an estimator that they is consistent under those conditions. CH study also the asymptotic properties of their estimator and characterize its large sample distribution. The estimator based on our median uncorrelation assumption is the same as the one used in CH. Finally, Sakata (2007) provides estimators based on an $\mathcal{L}_{1}$ loss function for instrumental regression models.

In Section 2, we provide first a few elementary definitions that lead to median uncorrelation. After defining median uncorrelation, Section 3 characterizes this uncorrelation concept in terms of various properties of the joint distribution of random variables. Section 4 shows how median uncorrelation leads to natural estimators in 
linear models with endogenous regressors. This notion is extended in section 5 to linear quantile regression with measurement error and to panel data quantile regression. Section 6 concludes.

\section{Definition AND Properties}

Let $T$ be a scalar random variable and let $S$ be a $k$-dimensional random vector such that $E|T|<\infty$ and $E|| S||<\infty$. We are interested in the following optimization problem since it is key in defining our concept of median uncorrelation:

$$
\min _{(\alpha, \beta)} E\left|T-\alpha-S^{\prime} \beta\right| .
$$

Define $M(T, S) \subset \mathcal{R}^{k}$ as the set of solutions to this problem with respect to $\beta$ :

$$
M(T, S) \equiv\left\{\beta: \exists \alpha \text { such that }(\alpha, \beta)=\underset{(\tilde{\alpha}, \tilde{\beta})}{\operatorname{argmin}} E\left|T-\tilde{\alpha}-S^{\prime} \tilde{\beta}\right|\right\} .
$$

In general, one can find distributions in which $M(T, S)$ is a set. However, under weak conditions, $M(T, S)$ is a singleton; see Propositions 1 and 2 below. Notice that for a fixed $\beta$,

$$
E\left|T-S^{\prime} \beta-\operatorname{Med}\left(T-S^{\prime} \beta\right)\right|=\min _{\alpha} E\left|T-\alpha-S^{\prime} \beta\right|,
$$

where

$$
\operatorname{Med}(z) \equiv \inf \{t: P(z \geq t) \geq 0.5\}
$$

Therefore,

$$
M(T, S)=\underset{\beta}{\operatorname{argmin}} E\left|T-S^{\prime} \beta-M e d\left(T-S^{\prime} \beta\right)\right| .
$$

The next propositions are important for deriving characterizations of median uncorrelation. Proposition 1 gives sufficient conditions that guarantee that $M(T, S)$ is a singleton. Proposition 2 provides a converse result.

Proposition 1. Suppose that equation

$$
E\left[S \operatorname{sgn}\left(T-S^{\prime} b-M e d\left(T-S^{\prime} b\right)\right)\right]=0
$$

has a unique solution ${ }^{1} b^{*}$. Then $M(T, S)$ is a singleton and $M(T, S)=b^{*}$.

\footnotetext{
${ }^{1}$ The function $\operatorname{sgn}(\cdot)$ is defined in the following way:

$$
\operatorname{sgn}(x)= \begin{cases}1, & x>0 \\ 0, & x=0 \\ -1, & x<0\end{cases}
$$
}


The proposition below establishes that if (2.1) has a solution, then the converse to the result in proposition 1 is also true. So, the combination of the those two propositions provide necessary and sufficient conditions for characterizing $M(T, S)$ as the solution to (2.1).

Proposition 2. Suppose that (2.1) has a solution. If $M(T, S)$ is a singleton, then $M(T, S)$ is the unique solution to (2.1).

The next definition introduces the notion of median uncorrelation of a random vector with another random vector. Here, and in the remainder of the paper, we take $M(T, S)=0$ to mean that $M(T, S)$ contains the single value 0 .

Definition 2.1 (Median Uncorrelation). Let $W$ denote an $l$-dimensional random vector. We will say that $W$ is median uncorrelated with $S$ if

$$
M\left(c^{\prime} W, S\right)=0 \text { for all } c \in \Re^{l} .
$$

The definition above is loss function based. So, it naturally carries over to quantiles other than median, by simply changing the absolute loss to asymmetric loss by using the "check function." Moreover, implicit in this definition, is a formulation for multivariate quantiles. In particular, when defining this uncorrelation property meant for scalar quantiles to the multivariate case, we require that median uncorrelation holds for any linear combination of the elements of the multivariate vector, as in (2.2). Finally, a key property that this "loss" function maintains is the invariance property below.

Lemma 2.1 (Invariance). For any constant vector $b \in \Re^{k}$ and any constant scalar $a$

$$
M\left(T+a+S^{\prime} b, S\right)=M(T, S)+b
$$

This property plays a key role below. Linearity of the model is essential for this invariance property to hold. The concept of uncorrelation we introduced itself, is intimately tied to the linear model and is similar to the relationship between uncorrelation in the least squares setup and its relationship to the linear model. Median uncorrelation is median linear uncorrelation. 


\section{Characterizations of Median Uncorrelation}

In this section, we provide key insights that explore further the meaning of median uncorrelation in definition 2.1 above. The following characterization lemma collects a set of properties that are helpful in gaining intuition about median uncorrelation.

Theorem 3.1 (Properties of Median Uncorrelation). The following hold:

A. A sufficient condition for an l-dimensional random vector $W$ to be medianuncorrelated with a random vector $S$ is that $M e d\left(c^{\prime} W \mid s\right)=\operatorname{Med}\left(c^{\prime} W\right)$ for all $c \in \Re^{l}$.

B. If $W$ is median-uncorrelated with $S$, it does not necessarily follow that $S$ is median-uncorrelated with $W$.

C. A sufficient condition for $W$ to be median uncorrelated with $S$ is that the conditional characteristic function of $W$ given $S$ is real.

D. Consider a scalar random variable $T$ and any random vector $S$. Assume that $M(T, S)$ is a singleton. Then $T$ can be written as

$$
T=\alpha_{0}+S^{\prime} M(T, S)+\delta,
$$

where $M(\delta, S)=0$, and $\alpha_{0}$ is any constant.

E. For a scalar random variable $T$ and random vectors $S$ and $Z$ in $\Re^{k}$, assume that equation (2.1) and the equation

$$
E\left[Z \operatorname{sgn}\left(T-Z^{\prime} b-\operatorname{Med}\left(T-Z^{\prime} b\right)\right)\right]=0
$$

have solutions, and $M(T, S+Z)$ is a singleton. Then,

$$
M(T, S)=M(T, Z)=0 \quad \Rightarrow \quad M(T, S+Z)=0 .
$$

F. For a scalar random variable $T$ and a binary $0 / 1$ random variable $S$ such that the median of $T \mid S=1$ and the median of $T \mid S=0$ are unique, the following hold:

if (2.1) has a solution, then

$M(T, S)=0 \quad \Longrightarrow \quad \operatorname{Med}(T \mid S=1)=\operatorname{Med}(T \mid S=0)=\operatorname{Med}(T) ;$

if $M(T, S)$ is a singleton, then

$$
M(T, S)=0 \Longleftarrow \operatorname{Med}(T \mid S=1)=\operatorname{Med}(T \mid S=0)=\operatorname{Med}(T) .
$$

Property (A) can be directly derived from the definition and basically states median independence as a sufficient condition for median uncorrelation. (B) is simple 
and is in direct contrast with mean uncorrelation which is a symmetric property. Property (D) is important and it states that any scalar random variable $T$ can be decomposed as a linear combination of $S$ 's and another random variable that is median uncorrelated with $S$. This is a direct result of the invariance property in (2.3) above. Moreover, this is similar to the linear mean decomposition in best linear prediction examples. See (3.1) below. Evidently, if $W$ is median-uncorrelated with $S$, then $S$ is not useful in the $\mathcal{L}_{1}$ prediction of linear functions of $W$.

3.1. Comparison to Mean Uncorrelation. It is helpful to compare the median uncorrelation with the usual mean uncorrelation, which is well known.

Consider the optimization problem

$$
\left.\min _{(\alpha, \beta)} E\left(T-\alpha-S^{\prime} \beta\right)^{2}\right) .
$$

This problem always has a unique solution. Denote its solution with respect to $\beta$ as $L(T, S)$. This is the $\mathcal{L}_{2}$ analogue of $M(T, S)$.

It is easy to show that, for scalar $S$, for example, $L(T, S)=\operatorname{Cov}(T, S) \operatorname{Var}(S)^{-1}$. In addition, $W$ and $S$ are (mean) uncorrelated if, for any $c \in \Re^{l}, c \neq 0, L\left(c^{\prime} W, S\right)=0$ since

$$
L\left(c^{\prime} W, S\right)=\operatorname{Var}(S)^{-1} \operatorname{Cov}(S, W) c
$$

Properties in Theorem 3.1 above have the following $\mathcal{L}_{2}$ versions.

$\mathcal{L}_{2}$ Properties. The following hold:

A. A sufficient condition for an $l$-dimensional random vector $W$ to be (mean) uncorrelated with a $k$-dimensional random vector $S$ is that $E\left(c^{\prime} W \mid S\right)=E\left(c^{\prime} W\right)$ for all $c \in \Re^{l}$. This holds in particular if $W$ is mean independent with $S$.

B. If $W$ is uncorrelated with $S$, then $S$ is uncorrelated with $W$.

C. A sufficient condition for $W$ to be uncorrelated with $S$ is that the conditional characteristic function of $W$ given $S$ is real.

D. For a scalar random variable $T$ and a $k$-dimensional random vector $S$, variable $T$ can be represented as follows:

$$
T=\alpha_{0}+S^{\prime} L(T, S)+\delta^{*}
$$

where $L\left(\delta^{*}, S\right)=0$ and $\alpha_{0}$ is any constant.

Clearly, If $W$ is uncorrelated with $S$, then $S$ is not useful in the $\mathcal{L}_{2}$ prediction of linear functions of $W$. 
The main technical differences between median uncorrelation and uncorrelation are that (1) median uncorrelation is not symmetric, (2) if $W_{1}$ and $W_{2}$ are both uncorrelated with $S$, then the vector $\left(W_{1}, W_{2}\right)$ is uncorrelated with $S$, while the same is not true for median uncorrelation, (3) a condition for $W$ and $S$ to be uncorrelated can be given in terms of $W$ alone (i.e., $\operatorname{Cov}(W, S)=0$ ) without reference to linear functions and (4) the additivity of $L(W, S)$, i.e., $L\left(W_{1}+W_{2}, S\right)=L\left(W_{1}, S\right)+L\left(W_{2}, S\right)$, which often greatly simplifies technical arguments. This latter difference basically means that if $W_{1}$ is uncorrelated with $S$ and $W_{2}$ is uncorrelated with $S$, then $W_{1}+W_{2}$ is uncorrelated with $S$. Two simple results in proposition 3 below compare the median uncorrelation with the usual mean uncorrelation.

Proposition 3. Let $T$ be a scalar random variable and $S$ be a random vector in $\mathcal{R}^{k}$.

(1) If $V$, a scalar random variable, is independent of $S$, then

$$
\operatorname{cov}(T+V, S)=\operatorname{cov}(T, S)
$$

but

$$
M(T+V, S) \neq M(T, S) \quad \text { in general. }
$$

(2) If $V$, a random vector in $\mathcal{R}^{k}$, is independent of $T$, then

$$
\operatorname{cov}(T, S+V)=\operatorname{cov}(T, S),
$$

but in general,

$$
M(T, S+V) \neq M(T, S)
$$

In the next section, we give a simple measure for median correlation that is bounded between -1 and 1 .

3.2. Measures of median correlation. In the case when two random variables are not median uncorrelated, we would like to be able to measure the degree of their median correlation. Two such measures are introduced below. The first generalizes the usual (mean) correlation; the second generalizes the idea of the coefficient of determination.

First, we review the $\mathcal{L}_{2}$ case. For scalar random variables $T$ and $S$, introduce the normalized random variables

$$
\begin{aligned}
& \tilde{T}=\frac{T-E(T)}{\sigma_{T}}, \\
& \tilde{S}=\frac{S-E(S)}{\sigma_{S}} .
\end{aligned}
$$


Correlation between $T$ and $S$ is measured by the correlation coefficient $\operatorname{corr}(T, S)$ :

$$
\operatorname{corr}(T, S)=E[|\tilde{T}||\tilde{S}| \operatorname{sgn}(\tilde{T}) \operatorname{sgn}(\tilde{S})]
$$

A second way to measure the linear relationship between two scalar random variables is to consider the extent to which a linear function of one random variable is useful in prediction of the other; when applied to data, this measure is the coefficient of determination, often denoted by $R^{2}$. Thus, let

$$
R^{2} \equiv r s q(T, S)=1-\frac{\min _{(\alpha, \beta)} E\left[(T-\alpha-\beta S)^{2}\right]}{E\left[(T-E(T))^{2}\right]} .
$$

It is well-known that $r s q(T, S)=\operatorname{corr}(T, S)^{2}$.

Now, consider the $\mathcal{L}_{1}$ case; we begin by considering the analogue of corr. Let

$$
\tilde{T}=\frac{T-\operatorname{Med}(T)}{E|T-\operatorname{Med}(T)|}
$$

and

$$
\tilde{S}=\frac{S-\operatorname{Med}(S)}{E|S-\operatorname{Med}(S)|} .
$$

Let medcorr $(T, S)$ be the measure of median correlation between $T$ and $S$. It is defined as:

$$
\operatorname{med} \operatorname{corr}(T, S) \equiv E[|\tilde{S}| \operatorname{sgn}(\tilde{T}) \operatorname{sgn}(\tilde{S})]
$$

Note that in general, medcorr $(T, S)$ is different from $M(T, S)$.

Theorem 3.2. For random variables $T$ and $S$, the following hold:

(1) $\operatorname{medcorr}(T, S) \in[-1,1]$.

(2) Suppose that the conditions in proposition 1 are satisfied. Then

$$
\operatorname{sgn}(\operatorname{medcorr}(T, S))=\operatorname{sgn}(M(T, S)) .
$$

Blomqvist (1950) introduced the following measure of median correlation between random variables $T$ and $S$ :

$$
k(T, S)=E[\operatorname{sgn}(T-M e d(T)) \operatorname{sgn}(S-\operatorname{Med}(S))],
$$

or, in terms of normalized variables

$$
k(T, S)=E[\operatorname{sgn}(\tilde{T}) \operatorname{sgn}(\tilde{S})] .
$$

As we can see, this measure is different from ours. In particular, $k(T, S)$ is symmetric and does not satisfy the invariance property. The value of $\operatorname{med} \operatorname{corr}(T, S)$ measures 
the degree of linear relationship between $T$ and $S$ while $k(T, S)$ represents an analog of Kendall's rank correlation because

$$
k(T, S)=\operatorname{Pr}((T-M e d(T))(S-M e d(S))>0)-\operatorname{Pr}((T-M e d(T))(S-M e d(S))<0) .
$$

The $\mathcal{L}_{1}$ analogue of $r s q$ is

$$
\operatorname{medrsq}(T, S) \equiv 1-\frac{\min _{\beta} E|T-\beta S-M e d(T-\beta S)|}{E|T-\operatorname{Med}(T)|} .
$$

Note that

$$
\operatorname{medrsq}(T, S)=1-\frac{E\left|T-\beta_{0} S-\operatorname{Med}\left(T-\beta_{0} S\right)\right|}{E|T-\operatorname{Med}(T)|},
$$

where $\beta_{0}$ is an arbitrary element of $M(T, S)$. This method was used in Koenker and Machado (1999) to measure the goodness of fit for quantile regressions. Koenker and Machado (1999) explain why medrsq is bounded between 0 and 1 . They also show that this correlation measure takes the value of 1 where the random variable $T$ and the random vector $S$ are linearly perfectly correlated.

We collect some results about medrsq and about the relationship between medcorr and medrsq in the following theorem.

Theorem 3.3. For random variables $T$ and $S$, the following hold

(1) If $M(T, S)=0$ then medrsq $(T, S)=0$; if medrsq $(T, S)=0$ then $0 \in$ $M(T, S)$.

(2) $\operatorname{medrsq}(T, S)=0$ if and only if $\operatorname{medcorr}(T, S)=0$.

Part (1) shows that medrsq takes the value of zero when $T$ is median uncorrelated with $S$. This is similar to the usual $R^{2}$ in linear models. Part (2) says that this median $R^{2}$ is equal to zero when the median correlation is zero.

Next, we generalize the concept of $\mathcal{L}_{1}$-correlation to other loss functions. This will be a natural extension to the above results.

3.3. $\mathcal{L}_{p}$-correlation for any $p \geq 1$. The notion of $\mathcal{L}_{1}$-correlation can be generalized to the case of $\mathcal{L}_{p}$-correlation for any $p \geq 1$.

Definition 3.1. For a random variable $Y$ and for any $p, 1 \leq p<\infty$, define $\operatorname{Med}_{p}(Y)$ as follows:

$$
\operatorname{Med}_{p}(Y) \equiv \inf \left\{d: E\left[|Y-d|^{p-1} \operatorname{sgn}(Y-d)\right]=0\right\}
$$


Note that $\operatorname{Med}_{1}(Y)=\operatorname{Med}(Y)$ and $\operatorname{Med}_{2}(Y)=E(Y)$.

Let $T$ be a random variable and $S$ be a random vector with values in $\Re^{k}$. Consider the optimization problem

$$
\min _{(\alpha, \beta)} E\left|T-\alpha-S^{\prime} \beta\right|^{p} .
$$

We are interested in the solutions to this problem with respect to $\beta$. Denote the set of these solutions as $M_{p}(T, S)$ :

$$
M_{p}(T, S) \equiv\left\{\beta: \exists \alpha \text { such that }(\alpha, \beta)=\underset{(\tilde{\alpha}, \tilde{\beta})}{\operatorname{argmin}} E\left|T-\tilde{\alpha}-S^{\prime} \tilde{\beta}\right|^{p}\right\} .
$$

Notice that for a fixed $\beta$,

$$
E\left|T-S^{\prime} \beta-\operatorname{Med}_{p}\left(T-S^{\prime} \beta\right)\right|^{p}=\min _{\alpha} E\left|T-\alpha-S^{\prime} \beta\right|^{p} .
$$

Therefore,

$$
M_{p}(T, S)=\underset{\beta}{\operatorname{argmin}} E\left|T-S^{\prime} \beta-\operatorname{Med}_{p}\left(T-S^{\prime} \beta\right)\right|^{p} .
$$

We can obtain results analogous to the ones in propositions 1 and 2 .

Proposition 4. Suppose that equation

$$
E\left[S \mid T-S^{\prime} b-\operatorname{Med}_{p}\left(T-S^{\prime p-1} \operatorname{sgn}\left(T-S^{\prime} b-\operatorname{Med}_{p}\left(T-S^{\prime} b\right)\right)\right]=0\right.
$$

has a unique solution $b^{*}$. Then $M_{p}(T, S)$ is a singleton and $M_{p}(T, S)=b^{*}$.

The next proposition establishes that if (3.2) has a solution, then the claim converse to the one in proposition 4 is true.

Proposition 5. Suppose that (3.2) has a solution. If $M_{p}(T, S)$ is a singleton, then $M_{p}(T, S)$ is the unique solution to (3.2).

The proofs of propositions 4 and 5 are omitted because they are similar to the proofs of propositions 1 and 2 .

The next definition introduces the notion of the $\mathcal{L}_{p}$-uncorrelation of a random vector with another random vector.

Definition $3.2\left(\mathcal{L}_{p}\right.$-uncorrelation). Let $W$ denote a $l$-dimensional random vector. We will say that $W$ is $\mathcal{L}_{p}$-uncorrelated with $S$ if

$$
M_{p}\left(c^{\prime} W, S\right)=0 \quad \text { for all } \quad c \in \Re^{l} .
$$


To measure the $\mathcal{L}_{p}$-correlation of a scalar random variable $T$ with a scalar random variable $S$, let us normalize these variable and define $\tilde{T}$ and $\tilde{S}$ in the following way:

$$
\begin{aligned}
& \tilde{T}=\frac{T-\operatorname{Med}_{p}(T)}{\left(E\left|T-\operatorname{Med}_{p}(T)\right|^{p}\right)^{\frac{1}{p}}}, \\
& \tilde{S}=\frac{S-\operatorname{Med}_{p}(S)}{\left(E\left|S-\operatorname{Med}_{p}(S)\right|^{p}\right)^{\frac{1}{p}}} .
\end{aligned}
$$

Define the $\mathcal{L}_{p}$-correlation of $T$ with $S$ as follows:

$$
\operatorname{medcorr}_{p}(T, S)=E\left[|\tilde{S}||\tilde{T}|^{p-1} \operatorname{sgn}(\tilde{T}) \operatorname{sgn}(\tilde{S})\right] .
$$

The value of $\operatorname{medcorr}_{p}(T, S)$ always lies in the interval $[-1,1]$, and it can be shown that under conditions in proposition 4 ,

$$
\operatorname{sgn}\left(\operatorname{medcorr}_{p}(T, S)\right)=\operatorname{sgn}\left(M_{p}(T, S)\right) .
$$

Note that if $T=c_{1}+c_{2} S$ with probability 1 , for some $c_{2}>0$, then $\operatorname{medcorr}_{p}(T, S)=$ 1. Also, if $T=c_{1}+c_{2} S$ with probability 1 , for some $c_{2}<0$, then $\operatorname{medcorr}_{p}(T, S)=$ -1 . It is easy to see that medcorr $2(T, S)$ coincides with the familiar correlation coefficient $\operatorname{corr}(T, S)$.

The $\mathcal{L}_{p}$ analogue of medrsq is defined as follows:

$$
\operatorname{medr}_{s}(T, S) \equiv 1-\frac{\min _{\beta} E\left|T-\beta S-\operatorname{Med}_{p}(T-\beta S)\right|^{p}}{E\left|T-\operatorname{Med}_{p}(T)\right|^{p}}
$$

and obviously,

$$
\operatorname{medrsq}(T, S)=1-\frac{E\left|T-\beta_{0} S-M_{e d}\left(T-\beta_{0} S\right)\right|^{p}}{E\left|T-M_{p}(T)\right|^{p}},
$$

where $\beta_{0}$ is an arbitrary element of $M_{p}(T, S)$.

\section{Median Uncorrelation and Instrumental Regression}

This is the main section of the paper in which we exploit the median uncorrelation concept to define estimators for parameters in linear models with endogenous variables. The estimator (and the model) are defined via the uncorrelation assumption in the same way as some versions of 2SLS are defined from the mean uncorrelation.

Consider the following model:

$$
Y=\alpha_{0}+X^{\prime} \beta_{0}+\epsilon
$$

where $Y$ and $\epsilon$ are real-valued random variables, $X$ is a $k$-dimensional random vector with a positive definite covariance matrix, $\alpha_{0}$ is an unknown scalar parameter, and 
$\beta_{0}$ is an unknown slope vector. The parameter of interest is $\beta_{0}$. Assume that $\epsilon$ has median 0 , but that

$$
\operatorname{Med}(\epsilon \mid x) \neq 0
$$

where $\operatorname{Med}(. \mid$.$) denotes the conditional median. The problem here is that this con-$ ditional median is allowed to depend on $X$. There are many reasons for this type of "endogeneity" in economic models. Classical work on demand and supply analysis in linear (in parameter) models motivate many early works in linear models with mean restrictions where instrumental variables assumptions were used to eliminate least squares bias that arises from this endogeneity. See Theil (1953), Basmann (1957) and Amemiya (1985) and references therein. There are a set of papers that deal with endogeneity in linear (and nonlinear) quantile based models. See for example Amemiya (1981) for a 2 stage interpretation of the 2SLS, and Chernozhukov and Hansen (2005) for an approach to inference in quantile based models, both linear and nonlinear, in the presence of endogenous regressors.

Recall that the 2SLS strategy is based on finding an instrument vector $Z$ such that $E[Z \epsilon]=0$, and using this uncorrelation (moment) condition to derive a consistent estimator for $\beta$. In this section, we extend this intuition to median uncorrelation whereas we assume the presence of a random vector $Z$, which we call a vector of instruments, that obeys a median uncorrelation assumption (see Assumption A.1 below). This median uncorrelation property, similarly to its counterpart $E[Z \epsilon]=0$, leads naturally to a simple estimator for $\beta_{0}$.

Assumption A.1. Let there be a d-dimensional random vector $Z$ such that:

1. There exists a $k \times d$ constant matrix of full rank $\gamma$, with $d \geq k$, such that

$$
X=\gamma Z+\delta
$$

for some random vector $\delta$.

2. $(\delta, \epsilon)^{\prime}$ is median uncorrelated with $Z$.

First, we require that the dimension of $Z$ be at least equal to the dimension of $X$. This is the necessary condition for point identification. In the case where this condition fails, the model will partially identify $\beta_{0}$. The key assumption is part 2 of A.1 where we require that not only $\epsilon$ be median uncorrelated with $Z$ and $\delta$ be median uncorrelated with $Z$, but also that $(\delta, \epsilon)^{\prime}=(X-\gamma Z, \epsilon)^{\prime}$ be jointly median uncorrelated with $Z$ (since the fact that $M(\epsilon, Z)=0$ and $M(\delta, Z)=0$ does not imply that $(\delta, \epsilon)^{\prime}$ is median uncorrelated with $Z$.) 
Given assumption A.1, we are able to easily prove the following theorem, which constitutes the main result in this section.

Theorem 4.1 (Main Result). Consider the function

$$
\Psi(\beta)=M\left(Y-X^{\prime} \beta, Z\right) .
$$

Let assumption A.1 hold. Then

$$
\Psi(\beta)=0 \quad \Longleftrightarrow \quad \beta=\beta_{0} .
$$

Proof: Note that by assumption A.1, we have

$$
Y=\alpha_{0}+Z^{\prime} \gamma^{\prime} \beta_{0}+\delta^{\prime} \beta_{0}+\epsilon
$$

Let

$$
m \in M\left(Y-X^{\prime} \beta, Z\right)=M\left(\alpha_{0}+Z^{\prime} \gamma^{\prime}\left(\beta_{0}-\beta\right)+\delta^{\prime}\left(\beta_{0}-\beta\right)+\epsilon, Z\right) .
$$

By the invariance property in lemma 2.1, there exists $m_{0} \in M\left(\delta^{\prime}\left(\beta_{0}-\beta\right)+\epsilon, Z\right)$ such that

$$
m=\gamma^{\prime}\left(\beta_{0}-\beta\right)+m_{0} .
$$

Note that $\delta^{\prime}\left(\beta_{0}-\beta\right)=\left(\beta_{0}-\beta\right)^{\prime} \delta$. Hence, since $(\delta, \epsilon)^{\prime}$ is median uncorrelated with $Z, m_{0}=0$. It follows that $m=\gamma^{\prime}\left(\beta_{0}-\beta\right)$ and, hence, that

$$
\Psi(\beta)=\gamma^{\prime}\left(\beta-\beta_{0}\right) .
$$

Since $d \geq k$ and $\gamma$ is full column rank by assumption A.1, we have

$$
\Psi(\beta)=0 \Longleftrightarrow \beta=\beta_{0},
$$

which proves the theorem.

The theorem can be used as the basis for an estimation method for $\beta_{0}$. Note that in case we use the least squares function $L(.,$.$) instead of M(.,$.$) , we get exactly$ Basmann's interpretation of the 2SLS estimator of $\beta_{0}$. Moreover, note that the estimator that is based on the result in the theorem above is the same as the one used by Chernozhukov and Hansen (2005). Let $\hat{Y}$ denote an $n \times 1$ vector of realizations of $Y$, let $\hat{X}$ denote an $n \times k$ matrix of realizations of $X$ and let $\hat{Z}$ denote an $n \times d$ matrix of realizations of $Z$. Define $\widehat{M}(\hat{Y}, \hat{Z})$ to be the vector $c \in \Re^{d}$ that minimizes

$$
\sum_{j}\left|\hat{Y}_{j}-a-\hat{Z}_{j}^{\prime} c\right|
$$

when minimizing over $(a, c)$. Then, $\hat{\beta}$ is defined as the solution in $b$ to

$$
\widehat{M}(\hat{Y}-\hat{X} b, \hat{Z})=0 \text {. }
$$


$\hat{\beta}$ can be obtained, as in $\mathrm{CH}$, by minimizing

$$
\hat{\beta}=\underset{b \in \Re^{k}}{\operatorname{argmin}}\|\widehat{M}(\hat{Y}-\hat{X} b, \hat{Z})\|_{A}
$$

where $\|\cdot\|_{A}$ is the weighted by $A$ Euclidian norm.

It is interesting to note that the sufficient condition for identification in $\mathrm{CH}$ adapted to the linear model is (in our notation) that for all $Z$ the following has a unique solution at the true parameter $\beta_{0}$ :

$$
P\left(Y<\alpha_{0}+X^{\prime} \beta \mid Z\right)=E\left[1\left[Y<\alpha_{0}+X^{\prime} \beta\right] \mid Z\right]=\frac{1}{2}
$$

while our median uncorrelation condition requires that the moment condition

$$
E\left[Z \operatorname{sgn}\left(Y-X^{\prime} \beta-\operatorname{Med}\left(Y-X^{\prime} \beta\right)\right)\right]=0
$$

has a unique solution at $\beta_{0}$.

CH's condition above can be written as

$$
E\left[\operatorname{sgn}\left(Y-X^{\prime} \beta_{0}-\operatorname{Med}\left(Y-X^{\prime} \beta_{0}\right)\right) \mid Z\right]=0,
$$

which obviously implies (4.3) when it is calculated at $\beta_{0}$. Clearly, it is a conditional statement, as opposed to an unconditional statement. Of course the identification approach in $\mathrm{CH}$ applies to a much larger class of models, including nonlinear ones.

We next state the asymptotic distribution without any conditions and refer the reader to Chernozhukov and Hansen (2005) for details, and for ways to compute the estimator and its standard errors. Under the conditions in $\mathrm{CH}$, as $N \rightarrow \infty$, we have

$$
\sqrt{N}(\hat{\beta}-\beta) \stackrel{d}{\rightarrow} \mathcal{N}\left(0, C^{-1} D\left[C^{-1}\right]^{\prime}\right),
$$

where $C=E\left[f_{\epsilon}(0 \mid X, Z) X Z^{\prime}\right]$ and $D=\frac{1}{4} E\left[Z Z^{\prime}\right]$ and $\epsilon=y-\alpha_{0}-X^{\prime} \beta_{0}$.

4.1. Relationship to 2SLS assumptions. In the usual endogenous model

$$
\begin{gathered}
Y=\alpha_{0}+X^{\prime} \beta_{0}+\epsilon, \\
\operatorname{Cov}(\epsilon, X) \neq 0 .
\end{gathered}
$$

Here, a random vector $Z$ is an instrument if $\operatorname{Cov}(X, Z)$ and $\operatorname{Cov}(Z, Z)$ have full rank and $\operatorname{Cov}(Z, \epsilon)=0$, or $E[Z \epsilon]=0$ with a mean zero assumption on $\epsilon$.

Let $\gamma=\operatorname{Cov}(X, Z) \operatorname{Cov}(Z, Z)^{-1}$ and define $\delta=X-\gamma Z$. Then,

$$
X=\gamma Z+\delta
$$

Here $(\delta, \epsilon)^{\prime}$ is uncorrelated with $Z$ because $\delta$ is uncorrelated with $Z$ by construction and $\epsilon$ is uncorrelated with $Z$ by definition. This is not true in the median case, where 
we need to impose condition in part 2 in Assumption A.1 above. This is the key difference between the mean and the median formulations.

\section{Other Applications of Median Uncorrelation}

We provide two other applications of this median uncorrelation by mimicking implications of mean uncorrelation when dealing with measurement error in linear models under quantile restrictions, and in panel data models with quantile restrictions.

5.1. Quantile Regression with Measurement Error. We apply the idea of median uncorrelation to linear quantile regressions with classical measurement error in the regressors. In particular, consider the model

$$
Y=\alpha_{0}+X^{*^{\prime}} \beta_{0}+\epsilon, \quad \operatorname{Med}(\epsilon)=0
$$

where we assume that $M\left(\epsilon, X^{*}\right)=0$ or that $\epsilon$ is median uncorrelated with a $k$ dimensional random vector $X^{*}$. We do not observe $X^{*}$ directly, but we observe an error-ridden version of it, $X$, such that

$$
X=X^{*}+\nu
$$

where we assume that $M\left(\nu, X^{*}\right)=0$. We also observe $Y$. To remedy the identification problem that results from the measurement error, we follow the treatment of the linear model under the mean uncorrelation and use instruments. Let there exist a $d$-dimensional random vector $Z$ and a $k \times d$ constant matrix $\gamma$, with $d \geq k$, such that

$$
X^{*}=\gamma Z+\psi
$$

for some random vector $\psi$, and $M(\psi, Z)=0$. Then

$$
X=\gamma Z+\psi+\nu
$$

Given the results of the previous section, we can show the following result.

Theorem 5.1. For model (5.1) suppose that we observe $(Y, X)$ such that (5.2) holds with $M\left(\nu, X^{*}\right)=0$. Moreover, assume that $(\epsilon, \nu, \psi)$ is median uncorrelated with $Z$ and that $\gamma$ in (5.3) has full rank. Then,

$$
M\left(Y-X^{\prime} \beta, Z\right)=0 \Longleftrightarrow \beta=\beta_{0} .
$$


Comments: Note, that the requirements of the above model are that the vector $(\epsilon, \nu, \psi)$ is jointly median uncorrelated with $Z$. The real assumption here is that the vector of unobservables is required to be median uncorrelated with $Z$. In contrast, in the mean uncorrelation model, all that is required is for $\epsilon$ to be uncorrelated with $Z$ and that for $Z$ to be correlated with $X^{*}$ (with the usual rank conditions). So, again, as in the 2SLS generalization, it is the joint median uncorrelation that is needed.

5.2. Quantile Regression with Panel Data. We are interested in inference on $\beta_{0}$ in the following model:

$$
y_{i t}=x_{i t}^{\prime} \beta_{0}+\alpha_{i}+\epsilon_{i t}, \quad t=1,2,
$$

where $\alpha_{i}$ is the individual effect that is arbitrarily correlated with $\mathbf{x}_{i}=\left(x_{i 1}^{\prime}, x_{i 2}^{\prime}\right)^{\prime}$. Denote $\Delta y_{i}=y_{i 1}-y_{i 2}, \Delta x_{i}=x_{i 1}-x_{i 2}$ and $\Delta \epsilon_{i}=\epsilon_{i 1}-\epsilon_{i 2}$. Suppose that we have a data set of iid observations $\left(\mathbf{y}_{i}, \mathbf{x}_{i}\right)$ for $i=1, \ldots, N$, where $\mathbf{y}_{i}=\left(y_{i 1}, y_{i 2}\right)^{\prime}$. If we maintain the assumption that $\epsilon_{i}=\left(\epsilon_{i 1}, \epsilon_{i 2}\right)^{\prime}$ is median uncorrelated with $\mathbf{x}_{i}$, then

$$
\beta_{0}=M\left(\Delta y_{i}, \Delta x_{i}\right)
$$

for any $i$. Indeed, this follows from

$$
E\left|\Delta y_{i}-a-\Delta x_{i}^{\prime} \beta\right|=E\left|\Delta \epsilon_{i}-a-\Delta x_{i}^{\prime}\left(\beta-\beta_{0}\right)\right|
$$

and the definition of the median uncorrelation of the vector $\epsilon_{i}$ with $\mathbf{x}_{i}$. In fact, it is possible to relax median uncorrelation to requiring that the random variable $\Delta \epsilon_{i}$ to be median uncorrelated with $\Delta x_{i}$.

\section{Conclusion}

The paper considers the 2SLS estimator that is commonly used in econometrics for estimating regressions with endogenous variables. This estimator is based on the assumption that even though a regressor is correlated with the error, there exists an excluded exogenous regressor that is (linearly) uncorrelated with the error. This regressor is called an instrument. And so, 2SLS exploits implications of this (linear) uncorrelation between the instrument and the error in the main regression to obtain a consistent estimator for the slope. This paper tries to follow the same model, but uses median uncorrelation instead. This median uncorrelation is new to our knowledge and is exactly similar to mean uncorrelation, except that it uses the absolute loss function, as opposed to the squared loss function used with the mean. We characterize properties of two vectors that are linearly median uncorrelated and 
then provide a measure of median uncorrelation that is bounded between -1 and 1 . This is meant to mirror the typical correlation coefficient in linear models. We also provide counterparts to $R^{2}$ the coefficient of determination. Most importantly, we show that in a linear regression model where the regressors are correlated with the errors, a median uncorrelation assumption between a set of instruments and the error provides the basis for inference on the linear slope parameter $\beta$ that is akin to what the 2SLS approach does under mean uncorrelation. We apply this uncorrelation concept to other examples like linear models with measurement error and quantile restrictions, and panel data quantile models. 


\section{Appendix}

Proof of Proposition 1. Consider and $b \in \Re^{k}$ such that $b \neq b^{*}$. The proposition will be proved if we show that

$$
E\left|T-S^{\prime} b^{*}-\operatorname{Med}\left(T-S^{\prime} b^{*}\right)\right|<E\left|T-S^{\prime} b-\operatorname{Med}\left(T-S^{\prime} b\right)\right| .
$$

The following holds:

$$
\begin{aligned}
& E\left|T-S^{\prime} b^{*}-\operatorname{Med}\left(T-S^{\prime} b^{*}\right)\right| \\
& ={ }_{(1)} E\left[T \operatorname{sgn}\left(T-S^{\prime} b^{*}-\operatorname{Med}\left(T-S^{\prime} b^{*}\right)\right)\right] \\
& ={ }_{(2)} E\left[\left(T-S^{\prime} b-\operatorname{Med}\left(T-S^{\prime} b\right)\right) \operatorname{sgn}\left(T-S^{\prime} b^{*}-\operatorname{Med}\left(T-S^{\prime} b^{*}\right)\right)\right] \\
& ={ }_{(3)} E\left[\left|T-S^{\prime} b-\operatorname{Med}\left(T-S^{\prime} b\right)\right| \operatorname{sgn}\left(T-S^{\prime} b-\operatorname{Med}\left(T-S^{\prime} b\right)\right) \operatorname{sgn}\left(T-S^{\prime} b^{*}-\operatorname{Med}\left(T-S^{\prime} b^{*}\right)\right)\right] \\
& <_{(4)} E\left|T-S^{\prime} b-\operatorname{Med}\left(T-S^{\prime} b\right)\right| .
\end{aligned}
$$

(1) and (2) follow from (2.1) and the definition of median. (4) follows from the fact that $b^{*}$ is the unique solution to (2.1), and therefore,

$$
P\left(\operatorname{sgn}\left(T-S^{\prime} b-\operatorname{Med}\left(T-S^{\prime} b\right)\right) \operatorname{sgn}\left(T-S^{\prime} b^{*}-\operatorname{Med}\left(T-S^{\prime} b^{*}\right)\right)=-1\right)>0 .
$$

Proof of Proposition 2. Let $b^{*}=M(T, S)$. First of all, we want to show that $b^{*}$ solves (2.1). Suppose this is not so:

$$
E\left[S \operatorname{sgn}\left(T-S^{\prime} b^{*}-\operatorname{Med}\left(T-S^{\prime} b^{*}\right)\right)\right] \neq 0 .
$$

Let $\tilde{b}$ be a solution to (2.1). Similar to how we did it in the proof of proposition 1 , we can show that

$$
E\left|T-S^{\prime} \tilde{b}-\operatorname{Med}\left(T-S^{\prime} \tilde{b}\right)\right|<E\left|T-S^{\prime} b^{*}-\operatorname{Med}\left(T-S^{\prime} b^{*}\right)\right|,
$$

which contradicts the fact that $b^{*}=M(T, S)$. Thus, $b^{*}$ is a solution to (2.1).

Now let us show that $b^{*}$ is the unique solution to (2.1). Suppose (2.1) has another solution $\tilde{b}, \tilde{b} \neq b^{*}$. Then, again, using techniques in the proof of proposition 1, we can establish that $E\left|T-S^{\prime} \tilde{b}-\operatorname{Med}\left(T-S^{\prime} \tilde{b}\right)\right| \leq E\left|T-S^{\prime} b^{*}-\operatorname{Med}\left(T-S^{\prime} b^{*}\right)\right|$ as well as $E\left|T-S^{\prime} b^{*}-\operatorname{Med}\left(T-S^{\prime} b^{*}\right)\right| \leq E\left|T-S^{\prime} \tilde{b}-\operatorname{Med}\left(T-S^{\prime} \tilde{b}\right)\right|$. This implies that $E\left|T-S^{\prime} b^{*}-\operatorname{Med}\left(T-S^{\prime} b^{*}\right)\right|=E\left|T-S^{\prime} \tilde{b}-\operatorname{Med}\left(T-S^{\prime} \tilde{b}\right)\right|$, which contradicts the fact that $M(T, S)$ is a singleton.

\section{Proof of Lemma 2.1.}


We prove this lemma in two steps. In the first step we show that $M(T, S)+b \subset$ $M\left(T+a+S^{\prime} b, S\right)$. In the second step, we establish that $M\left(T+a+S^{\prime} b, S\right) \subset$ $M(T, S)+b$.

First of all, note that for a given $b$ and any $a$,

$$
M\left(T+a+S^{\prime} b, S\right)=\underset{q \in \Re^{k}}{\operatorname{argmin}} E\left|T+S^{\prime}(b-q)-\operatorname{Med}\left(T+S^{\prime}(b-q)\right)\right| .
$$

Let $m_{1} \in M(T, S)$. This implies that for any $q \in \Re^{k}$

$$
E\left|T+S^{\prime}(b-q)-\operatorname{Med}\left(T+S^{\prime}(b-q)\right)\right| \geq E\left|T-S^{\prime} m_{1}-\operatorname{Med}\left(T-S^{\prime} m_{1}\right)\right| .
$$

Obviously, the inequality becomes the equality if $q=m_{1}+b$. Therefore, $m_{1}+b \in$ $M\left(T+a+S^{\prime} b, S\right)$.

Now let $m_{2} \in M\left(T+a+S^{\prime} b, S\right)$. This implies that for any $\beta \in \Re^{k}$

$$
E\left|T-S^{\prime} \beta-\operatorname{Med}\left(T-S^{\prime} \beta\right)\right| \geq E\left|T+S^{\prime}\left(b-m_{2}\right)-\operatorname{Med}\left(T+S^{\prime}\left(b-m_{2}\right)\right)\right| .
$$

The inequality becomes the equality if $\beta=m_{2}-b$. Therefore, $m_{2}-b \in M(T, S)$ and, hence, $m_{2} \in M(T, S)+b$.

\section{Proof of Theorem 3.1.}

$(A)$ : Suppose $\operatorname{Med}\left(c^{\prime} W \mid s\right) \equiv c^{*}$. Then, we know that $c^{*}$ minimizes the following problem over ALL (measurable) functions $g(S)$ :

$$
E\left|c^{\prime} W-c^{*}\right| \leq E\left|c^{\prime} W-g(S)\right|
$$

In particular, this holds for any linear function of $S, \alpha+S^{\prime} \beta$ with $\beta \neq 0$.

$(B)$ : It is clear from the definition of median uncorrelation. This means that the definition of median uncorrelation is not symmetric.

$(C)$ : This means that the conditional characteristic function of $c^{\prime} W$ given $S$ is real, which in part means that the conditional distribution of $c^{\prime} W$ given $S$ is symmetric around 0. Hence, $\operatorname{Med}\left(c^{\prime} W \mid s\right)=0=\operatorname{Med}\left(c^{\prime} W\right)$ for all $s$.

$(D)$ : Let $\delta=T-\alpha_{0}-S^{\prime} M(T, S)$, where $\alpha_{0}$ is any constant. Showing that $M(\delta, S)$ is equal to 0 is a direct result of the invariance property in (2.3).

$(E)$ : Proposition 2 and the conditions $M(T, S)=0$ and $M(T, Z)=0$ imply that

$$
E[\operatorname{Ssgn}(T-M e d(T))]=0, \quad E[Z \operatorname{sgn}(T-M e d(T))]=0 .
$$

Then

$E[(S+Z) \operatorname{sgn}(T-M e d(T))]=E[S \operatorname{sgn}(T-M e d(T))]+E[Z \operatorname{sgn}(T-M e d(T))]=0$. 
The last equation and the assumption that $M(T, S+Z)$ is a singleton, and proposition 2 imply that $M(T, S+Z)=0$.

$(F)$ : For the first part of the statement, we note that proposition 2, the conditions that (2.1) has a solution and that $M(T, S)=0$ imply

$$
E[\operatorname{Ssgn}(T-\operatorname{Med}(T))]=0 .
$$

For the second part of the statement, we note that proposition 2, the equation $E[\operatorname{Sggn}(T-\operatorname{Med}(T))]=0$ and the fact that $M(T, S)$ is a singleton imply that $M(T, S)=0$.

The discussion below proves the statement.

Given that the conditional median of $T \mid S=1$ is unique, we have:

$$
\begin{aligned}
E[\operatorname{Ssgn}(T-\operatorname{Med}(T))]=0 & \Longleftrightarrow E[\operatorname{sgn}(T-\operatorname{Med}(T)) \mid S=1]=0 \\
& \Longleftrightarrow \operatorname{Med}(T)=\operatorname{Med}(T \mid S=1) .
\end{aligned}
$$

Because $E[\operatorname{sgn}(T-M e d(T))]=0$, then

$$
E[\operatorname{sgn}(T-M e d(T)) \mid S=1]=0 \quad \Longleftrightarrow \quad E[\operatorname{sgn}(T-\operatorname{Med}(T)) \mid S=0]=0 .
$$

Therefore, it is also true that

$$
E[S \operatorname{sgn}(T-\operatorname{Med}(T))]=0 \quad \Longleftrightarrow \quad \operatorname{Med}(T)=\operatorname{Med}(T \mid S=0) .
$$

\section{Proof of Theorem 3.2.}

(1): This follows from

$$
|\operatorname{medcorr}(T, S)|=|E[\tilde{S} \operatorname{sgn}(\tilde{T}) \operatorname{sgn}(\tilde{S})]| \leq E[|\tilde{S}|]=1
$$

(2): First, let us prove that $M(T, S)=0 \Longleftrightarrow \operatorname{medcorr}(T, S)=0$.

$$
\begin{aligned}
M(T, S)=0 & \Longleftrightarrow E[S \operatorname{sgn}(T-\operatorname{Med}(T))]=0 \\
& \Longleftrightarrow E\left[\frac{S-M e d(S)}{E|S-M e d(S)|} \operatorname{sgn}\left(\frac{T-M e d(T)}{E|T-M e d(T)|}\right)\right]=0 \\
& \Longleftrightarrow E[\tilde{S} \operatorname{sgn}(\tilde{T})]=0 \\
& \Longleftrightarrow \quad \operatorname{med} \operatorname{corr}(T, S)=0 .
\end{aligned}
$$

Note that $\operatorname{medcorr}(T, S)=\operatorname{medcorr}(\tilde{T}, \tilde{S})$ and

$$
M(T, S)=\frac{E|T-M e d(T)|}{E|S-M e d(S)|} M(\tilde{T}, \tilde{S}),
$$


and hence, $\operatorname{sgn}(M(T, S))=\operatorname{sgn}(M(\tilde{T}, \tilde{S}))$. Thus, it is enough to show that

$$
\operatorname{sgn}(\operatorname{medcorr}(\tilde{T}, \tilde{S}))=\operatorname{sgn}(M(\tilde{T}, \tilde{S}))
$$

Denote $b^{*}=M(\tilde{T}, \tilde{S})$. For $b^{*}=0$ the result is already proven.

Suppose $b^{*} \neq 0$. Notice that

$$
\operatorname{sgn}(\operatorname{medcorr}(\tilde{T}, \tilde{S}))=\operatorname{sgn}\left(b^{*}\right) \operatorname{sgn}\left(E\left[b^{*} \tilde{S} \operatorname{sgn}(\tilde{T})\right]\right),
$$

therefore, the result will be proven if we establish that $E\left[b^{*} \tilde{S} \operatorname{sgn}(\tilde{T})\right]>0$.

Indeed, using the fact that $b^{*}$ satisfies

$$
E\left[\tilde{S} \operatorname{sgn}\left(\tilde{T}-b^{*} \tilde{S}-\operatorname{Med}\left(\tilde{T}-b^{*} \tilde{S}\right)\right)\right]=0,
$$

we obtain

$$
\begin{aligned}
E\left[b^{*} \tilde{S} \operatorname{sgn}(\tilde{T})\right] & =E\left[\left(b^{*} \tilde{S}+\operatorname{Med}\left(\tilde{T}-b^{*} \tilde{S}\right)\right) \operatorname{sgn}(\tilde{T})\right] \\
& =E\left[\left(b^{*} \tilde{S}+\operatorname{Med}\left(\tilde{T}-b^{*} \tilde{S}\right)\right)\left(\operatorname{sgn}(\tilde{T})-\operatorname{sgn}\left(\tilde{T}-b^{*} \tilde{S}-\operatorname{Med}\left(\tilde{T}-b^{*} \tilde{S}\right)\right)\right]\right. \\
& =2 E\left[\left(b^{*} \tilde{S}+\operatorname{Med}\left(\tilde{T}-b^{*} \tilde{S}\right)\right) 1(\tilde{T}>0) 1\left(\tilde{T}-b^{*} \tilde{S}-\operatorname{Med}\left(\tilde{T}-b^{*} \tilde{S}\right)<0\right)\right] \\
& -2 E\left[\left(b^{*} \tilde{S}+\operatorname{Med}\left(\tilde{T}-b^{*} \tilde{S}\right)\right) 1(\tilde{T}<0) 1\left(\tilde{T}-b^{*} \tilde{S}-\operatorname{Med}\left(\tilde{T}-b^{*} \tilde{S}\right)>0\right)\right] \\
& +E\left[|\tilde{T}| 1\left(\tilde{T}=b^{*} \tilde{S}+\operatorname{Med}\left(\tilde{T}-b^{*} \tilde{S}\right)\right)\right] \\
& +E\left[\left|b^{*} \tilde{S}+\operatorname{Med}\left(\tilde{T}-b^{*} \tilde{S}\right)\right| 1(\tilde{T}=0)\right]
\end{aligned}
$$

Notice that all the four terms in the last sum are non-negative. Moreover, at least one of the first two terms in this sum is strictly positive because

$$
\operatorname{Pr}\left(\operatorname{sgn}(\tilde{T}) \operatorname{sgn}\left(\tilde{T}-b^{*} \tilde{S}-\operatorname{Med}\left(\tilde{T}-b^{*} \tilde{S}\right)\right)=-1\right)>0,
$$

which can be proven by applying techniques from the proof of proposition 1 and taking into account that $b^{*} \neq 0$. Therefore, $E\left[b^{*} \tilde{S} \operatorname{sgn}(\tilde{T})\right]>0$.

Proof of Theorem 3.3.

(1): If $M(T, S)=0$, then

$$
\operatorname{medrsq}(T, S)=1-\frac{E|T-M e d(T)|}{E|T-M e d(T)|}=0 .
$$

If $\operatorname{medrsq}(T, S)=0$, then

$$
\min _{\beta} E|T-\beta S-M e d(T-\beta S)|=E|T-M e d(T)|,
$$

so that, clearly, $0 \in M(T, S)$. 
(2): If $\operatorname{medrsq}(T, S)=0$, then, from part (3), $0 \in M(T, S)$. It follows that $E[S \operatorname{sgn}(T-M e d(T))]=0$ and, hence, that $\operatorname{medcorr}(T, S)=0$. If $\operatorname{medcorr}(T, S)=$ 0 , then $E[S \operatorname{sgn}(T-M e d(T))]=0$ so that $0 \in M(T, S)$. It follows that

$$
\min _{\beta} E|T-\beta S-M e d(T-\beta S)|=E|T-\operatorname{Med}(T)| .
$$

\section{Proof of Theorem 5.1.}

The proof of this theorem is analogous to the proof of theorem 4.1.

Let

$$
m \in M\left(Y-X^{\prime} \beta, Z\right)=M\left(\alpha_{0}+Z^{\prime} \gamma^{\prime}\left(\beta_{0}-\beta\right)+\psi^{\prime}\left(\beta_{0}-\beta\right)+\epsilon-\nu^{\prime} \beta, Z\right) .
$$

By the invariance property in lemma 2.1, there exists $m_{0} \in M\left(\psi^{\prime}\left(\beta_{0}-\beta\right)+\epsilon-\nu^{\prime} \beta, Z\right)$ such that

$$
m=\gamma^{\prime}\left(\beta_{0}-\beta\right)+m_{0} .
$$

Note that $\psi^{\prime}\left(\beta_{0}-\beta\right)=\left(\beta_{0}-\beta\right)^{\prime} \psi$ and $\nu^{\prime} \beta=\beta^{\prime} \nu$. Hence, since $(\epsilon, \nu, \psi)^{\prime}$ is median uncorrelated with $Z, m_{0}=0$. It follows that $m=\gamma^{\prime}\left(\beta_{0}-\beta\right)$, and hence, that

$$
M\left(Y-X^{\prime} \beta, Z\right)=\gamma^{\prime}\left(\beta_{0}-\beta\right) .
$$

Since $d \geq k$ and $\gamma$ is full column rank by assumption, then

$$
M\left(Y-X^{\prime} \beta, Z\right)=0 \Longleftrightarrow \beta=\beta_{0} .
$$




\section{REFERENCES}

Amemiya, T. (1981): "Two Stage Least Absolute Deviations Estimators," Econometrica, 50, 689711.

(1985): Advanced Econometrics. Harvard University Press.

Basmann, R. (1957): "A Generalized Classical Method of Linear Estimation of Coefficients in a Structural Equation," Econometrica, 25, 77-83.

Blomqvist, N. (1950): "On a Measure of Dependence Between two Random Variables," The Annals of Mathematical Statistics, 21(4), 593-600.

Chernozhukov, V., And C. Hansen (2005): "An IV Model of Quantile Treatment Effects," Econometrica, 73, 245-261.

Honoré, B., AND L. Hu (2004): "On the Performance of Some Robust Instrumental Variables Estimators," Journal of Business and Economic Statistics, 22(1), 30-39.

Koenker, R. (2005): Quantile Regression. Cambridge University Press.

Koenker, R., And G. Bassett (1978): "Regression Quantiles," Econometrica, 46, 33-50.

Koenker, R., And J.A.F. Machado (1999): "Goodness of Fit and Related Inference Processes for Quantile Regression," Journal of the American Statistical Association, 94(448), 1296-1310.

Manski, C. F. (1988): Analog Estimation Methods in Econometrics. Chapman and Hall.

SAKATA, S. (2007): "Instrumental variable estimation based on conditional median restriction," Journal of Econometrics, 141(2), 350-382.

Theil, H. (1953): "Estimation and Simultaneous Correlation in Complete Equation Systems," The Hague: Centraal Planbureau. 